\title{
Nuevos anticoagulantes orales
}

\author{
ALEJANDRO BERKOVITS ${ }^{1}$, ANDRÉS AIZMAN², PAMELA ZÚÑIGA ${ }^{1}$, \\ JAIME PEREIRA ${ }^{1}$, DIEGO MEZZANO ${ }^{1}$
}

\section{New oral anticoagulant drugs}

Thromboembolic disease (TED) is the leading cause of morbidity and mortality worldwide. The hallmark of oral long-term anticoagulant therapy has been the use of vitamin K antagonists, whose anticoagulant effect is exerted inhibiting vitamin $K$ epoxide reductase. Warfarin and acenocoumarol are the most commonly used. In the last five years several new drugs for long term anticoagulation have been developed, which can inhibit single clotting factors with the purpose of improving drug therapeutic range and, ideally, minimizing bleeding risks. This review addresses the state of the art on the clinical use of inhibitors of activated factor $X$ and thrombin.

(Rev Med Chile 2011; 139: 1347-1355).

Key words: Anticoagulants; Haemostasis; Thrombosis.

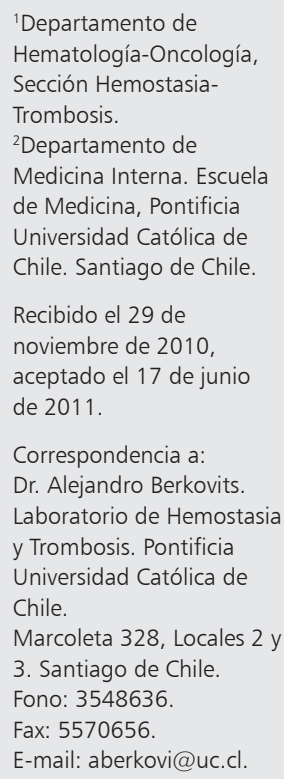

I

a anticoagulación oral tuvo su génesis en 1941. Karl Paul Link, de la Universidad de Jisconsin, logró aislar dicumarol desde cumarina, una molécula derivada de una planta dulce llamada "hierba del bisonte" que producía sangrado en el ganado que la comía. Este nuevo compuesto desarrollado recibió el nombre de Warfarin (Wisconsin Alumni Research Foundation, y arin de coumarin $)^{1}$. En 1978 se describió su mecanismo de acción anticoagulante, inhibiendo la activación de la vitamina $\mathrm{K}^{2}$.

Inicialmente usado como raticida, desde el año 1950 hasta la fecha, estos fármacos han sido el pilar del tratamiento anticoagulante oral, siendo ampliamente utilizados en la prevención y el tratamiento de la enfermedad tromboembólica arterial y venosa.

Los inhibidores de vitamina $\mathrm{K}$ tienen limitaciones. La principal es el estrecho rango terapéutico, requiriendo exámenes periódicos para ajuste de dosificación y monitorización del efecto anticoagulante. Para ello se utiliza el INR (International Normalized Ratio), considerándose apropiado en la mayoría de los casos un INR entre 2 y 3.

Los pacientes con rangos de anticoagulación bajo el nivel terapéutico presentan riesgo de trombosis, mientras que con niveles superiores al normal, la probabilidad de presentar sangrado patológico puede llegar a más de 30\% al año (INR mayor a 5$)^{3}$.

Es por eso que se están desarrollando nuevos anticoagulantes con farmacocinética y farmacodinamia más predecibles, mayor rango terapéutico y dosis fija que no requiera monitorización. Estos se han enfocado en la inhibición específica del factor $\mathrm{X}$ activado (FXa) y de la trombina (IIa), enzimas claves en la vía final de la coagulación ${ }^{4}$.

\section{Farmacofisiología}

La hemostasia es un proceso dinámico cuyo objetivo es la mantención de la integridad de los vasos sanguíneos y la sangre circulando en fase líquida. Una activación patológica del proceso, con generación excesiva de trombina, inicia el fenómeno de trombosis formando fibrina con obstrucción del lumen vascular. Este es el evento crítico que determina la alta morbilidad y mortalidad de esta patología.

El compromiso arterial está asociado principalmente a infarto del miocardio y accidente cerebrovascular oclusivo, siendo estas las causas más frecuentes de mortalidad mundial y nacional. ${ }^{5}$ En Chile, en el año 2009, de los 91.965 fallecidos, $27 \%$ correspondieron a enfermedades del sistema 
circulatorio, siendo estas principalmente trombosis coronaria y cerebrovascular ${ }^{6}$.

La enfermedad tromboembólica venosa constituye la principal causa de morbimortalidad prevenible en pacientes hospitalizados y es la segunda causa de muerte en aquellos con cáncer ${ }^{7}$.

Es por eso que entender la patogenia de la trombosis es fundamental para detectar posibles blancos terapéuticos con el consiguiente desarrollo de nuevos fármacos para su prevención y tratamiento.

La ruptura vascular, el daño endotelial o los accidentes de placa ateromatosa producen exposición de la matriz subendotelial, estimulando la adhesión plaquetaria mediada por integrina $\mathrm{a}_{2} \mathrm{~b}_{1} \mathrm{y}$ glicoproteína VI (GPVI) a colágeno y glicoproteína Ib-V-IX a factor von Willebrand (FVW). Con esto se origina una activación plaquetaria con la subsecuente secreción granular y agregación. El tapón plaquetario inicial provee la superficie rica en fosfolípidos aniónicos en donde se ensamblan los complejos macro-moleculares y se efectúan las reacciones enzimáticas que concluyen en la generación de trombina ${ }^{8}$.

La exposición del factor tisular (FT), ya sea desde el subendotelio (pared vascular o ateroma) o desde células circulantes que lo pueden expresar (ej.: monocitos en las sepsis), activa el Factor VII (FVIIa). El complejo resultante, FT/VIIa, cataliza la activación de los factores X y IX. Estos tienen funciones disímiles en la iniciación de la coagulación.
El FXa interactúa con su cofactor Va sobre superficies fosfolipídicas formando el complejo protrombinasa y genera pequeñas cantidades de trombina (IIa). El IXa, si bien no está involucrado de manera directa en la iniciación de la coagulación, en caso de daño vascular y formación de tapón plaquetario, puede difundir hacia la superficie de plaquetas activadas uniéndose a su receptor específico e interactúa con su cofactor, FVIIIa (formando el complejo tenasa) activando más cantidad de FXa.

La trombina generada por el FXa potencia la formación del tapón plaquetario y, por un proceso de retro-alimentación positiva, activa los factores XI, XIII, VIII y V.

El sustrato del FXIa es el FIX, y el complejo FIXa-VIIIa mantiene la cadena de formación de FXa-FVa, el que a su vez gatilla la producción masiva de trombina con formación del coágulo de fibrina a partir del fibrinógeno circulante $e^{9-12}$.

Los anticoagulantes actúan en diversos puntos de la cascada de la coagulación (Figura 1).

\section{Anticoagulantes tradicionales}

\section{Antagonistas de la vitamina $K$}

La vitamina $\mathrm{K}$ en su forma reducida (hidroquinona) es un cofactor de la enzima hepática $\gamma$ glutamil-carboxilasa, que cataliza la carboxilación post-traslacional de varios residuos de glutamato a ácido $\gamma$-carboxiglutámico, (residuos Gla) en los

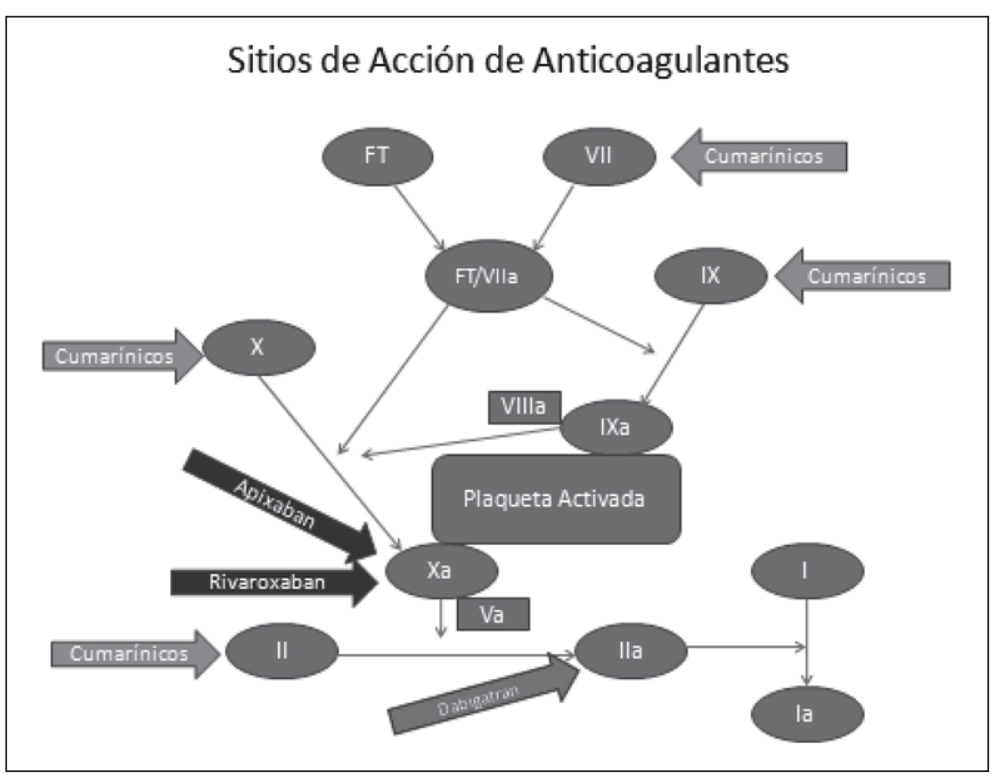

1348
Figura 1. Blanco terapéutico de los nuevos anticoagulantes. 
extremos NH-terminales de los factores II, VII, IX y X. Los residuos Gla ligan $\mathrm{Ca}++\mathrm{y}$ así se unen a fosfolípidos aniónicos en las membranas celulares; por este mecanismo, los residuos Gla confieren actividad biológica a los factores dependientes de la vitamina $\mathrm{K}$. El proceso de carboxilación oxida la vitamina $\mathrm{K}$ a su forma epóxido, que se reduce, reciclándose a su forma activa (hidroquinona) por el complejo de reductasas denominado vitamina $\mathrm{K}$ óxido reductasa 1 (VKORC 1). La acción de dichas reductasas es inhibida por los anticoagulantes orales antagonistas de la vitamina $\mathrm{K}^{13,14}$.

Los inhibidores de la vitamina $\mathrm{K}$ son anticoagulantes efectivos, con buena absorción y bajo costo. En caso de exceso de anticoagulación existen métodos para revertir su efecto. Las principales desventajas son la interacción con fármacos y alimentos que modifican su metabolismo generando variabilidad en un mismo paciente e inestabilidad dosis-respuesta entre distintos pacientes. Esto obliga a monitorizar el tratamiento con mediciones periódicas del INR.

\section{Warfarina}

Es el más utilizado a nivel mundial y en la mayoría de los estudios clínicos. Tiene un $\mathrm{T} 1 \frac{1}{2}$ de 35 a 45 horas.

\section{Acenocumarol}

El anticoagulante oral más usado en Chile. Su acción, ventajas y desventajas son similares a la warfarina, pero difiere de ésta por tener un $\mathrm{T}^{1} / 2$ más corto, de 8 a 24 horas, lo que podría significar una mayor inestabilidad del rango terapéutico. ${ }^{15}$

\section{Nuevos anticoagulantes orales (Tabla 1)}

\section{Inhibidores de la trombina}

Son antagonistas competitivos de la trombina capaces de inhibir su función, tanto en la fracción soluble como unida a fibrina. Esta propiedad de inhabilitar la trombina unida a fibrina los diferencia de las heparinas. Además, estas nuevas drogas afectan la activación por trombina de los factores V, VIII y XI y su propiedad de agonista plaquetario. Las principales ventajas son su uso en dosis fija siendo innecesaria la monitorización de sus niveles de anticoagulación ${ }^{16}$.

- Dabigatran etexilate: Prodroga. Inhibidor competitivo y reversible de la trombina. Requiere pH ácido para su absorción por lo que no se recomienda su ingesta en conjunto con inhibidores de la bomba de protones. Es convertida en el hígado a dabigatran (droga activa), sin utilizar citocromo p450 por lo que tiene baja interacción con otras drogas. Su biodisponibilidad es de 7,2\%. Tiene rápido inicio de acción, con máximo plasmático en 1,5-3 horas y T12 12 de 12 a 17 horas. Se excreta principalmente por el riñón $(80 \%)$, por lo que pacientes con insuficiencia renal han sido excluidos de la mayoría de los estudios ${ }^{17,18}$.

Tabla 1. Tabla comparativa de nuevos anticoagulantes

\begin{tabular}{|c|c|c|c|c|c|}
\hline Fármaco & $\begin{array}{l}\text { Mecanismo } \\
\text { acción }\end{array}$ & $\begin{array}{l}\text { Dosis } \\
\text { profilaxis }\end{array}$ & $\begin{array}{l}\text { Dosis } \\
\text { tratamiento }\end{array}$ & $\begin{array}{l}\text { Vida } \\
\text { media }\end{array}$ & $\begin{array}{l}\text { Aprobación basada } \\
\text { en evidencia }\end{array}$ \\
\hline Warfarina & $\begin{array}{l}\text { Inh. Epóxido } \\
\text { vitamina K reductasa }\end{array}$ & Según INR & Según INR & $\begin{array}{l}35-45 \\
\text { horas }\end{array}$ & $\begin{array}{l}\text { Profilaxis y Tratamiento } \\
\text { ETE }\end{array}$ \\
\hline Acenocumarol & $\begin{array}{l}\text { Inh. Epóxido } \\
\text { vitamina K reductasa }\end{array}$ & Según INR & Según INR & $\begin{array}{l}8-24 \\
\text { horas }\end{array}$ & $\begin{array}{l}\text { Profilaxis y tratamiento } \\
\text { ETE }\end{array}$ \\
\hline Dabigatran & Inh. Ila & $150-220 \mathrm{mg}$ QD & 110 mg o 150 mg BID & $\begin{array}{l}12-17 \\
\text { horas }\end{array}$ & $\begin{array}{l}\text { Profilaxis ETE } \\
\text { Tratamiento ETE en FA }\end{array}$ \\
\hline Rivaroxaban & Inh. Xa & $10 \mathrm{mg}$ QD & $\begin{array}{l}20 \text { mg QD (15 mg QD } \\
\text { en caso de insuficiencia } \\
\text { renal) }\end{array}$ & $\begin{array}{c}\text { 5-9 } \\
\text { horas }\end{array}$ & $\begin{array}{l}\text { Profilaxis ETE post ciru- } \\
\text { gía rodilla/cadera }\end{array}$ \\
\hline Apixaban & Inh. Xa & $2,5-5 \mathrm{mg}$ BID & & $\begin{array}{l}\text { 9-14 } \\
\text { horas }\end{array}$ & $\begin{array}{l}\text { Estudios fase III en } \\
\text { curso }\end{array}$ \\
\hline Betrixaban & Inh. Xa & 40-80 mgQD & & & Estudios Fase II en curso \\
\hline Edoxaban & Inh. Xa & 30-60 mg QD & & & Estudio fase III en curso \\
\hline
\end{tabular}




\section{Inhibidores de FXa}

Actúan en una etapa previa en la cascada de la coagulación. Inhiben al FXa libre, unido al complejo protrombinasa (junto a cofactor $\mathrm{V}$ ) y al FXa asociado a trombina. Al igual que los inhibidores de la trombina, no requieren monitorización de niveles de anticoagulación y se administran en dosis fija ${ }^{16}$.

- Rivaroxaban: Tiene 80\% de biodisponibilidad y, como dabigatran, un rápido inicio de actividad, alcanzando un máximo plasmático en 3 a 4 horas. Su T1/2 varía según la función renal, siendo en promedio de 5 a 9 horas en los jóvenes y 11 a 13 horas en adultos mayores, con clearance de creatinina $<$ a $50 \mathrm{ml} / \mathrm{min}$. La excreción es $70 \%$ renal y $30 \%$ hepática. Tiene baja interacción con otros fármacos, pero los azoles e inhibidores de proteasa-VIH pueden aumentar sus niveles plasmáticos ${ }^{19}$.

- Apixaban: Posee una biodisponibilidad mayor al 50\% con máximo plasmático de 3 horas y $\mathrm{T} 1 / 2$ de 12 . La metabolización es principalmente hepática, sin inducción o inhibición del sistema de citocromos, por lo que la interacción con otras drogas es mínima. La excreción es mayoritariamente fecal, con sólo $25 \%$ por vía renal ${ }^{20}$.

\section{Inhibidores de epóxido reductasa de vitamina $K$}

- Tecarfarina: Su T12/2 es de 119 horas. Su estructura es análoga a la warfarina, con alta unión a proteínas (99\%) y metabolizada por carboxilo-esterasas microsomales hepáticas, llevando a un metabolito inactivo, ATI-5900. La principal diferencia con otros inhibidores de vitamina $\mathrm{K}$ es que no es metabolizado por el sistema de citocromo p-450, disminuyendo las posibles interacciones con otros fármacos $\mathrm{y}$ alimentos ${ }^{21}$.

\section{Evidencia para el uso clínico de los nuevos anticoagulantes}

\section{Tromboembolismo arterial (Tabla 2)}

\section{Dabigatran}

El estudio RE-LY (Randomized Evaluation of Long Term Anticoagulation Therapy), publicado en 2009, evaluó a más de 18.000 individuos con fibrilación auricular (FA). Comparó dos dosis de dabigatran, $110 \mathrm{mg}$ dos veces al día (BID) y 150 mg (BID) con warfarina (INR 2-3) en prevención secundaria de fenómenos embólicos cerebrales y sistémicos. Se excluyeron pacientes con insuficiencia renal.

En seguimiento a dos años las tasas anuales de embolias fueron $1,53 \%$ con $110 \mathrm{mg}$ BID (bis in die $=$ dos veces al día) de dabigatran y $1,69 \%$ con warfarina (RR de 0,91 (95\% IC 0,74-1,11), con significación estadística para no-inferioridad. Con $150 \mathrm{mg}$ BID de dabigatran la incidencia de embolias fue de $1,11 \%$ que comparado con warfarina muestra una disminución del riesgo relativo de 44\% (RR 0,66 (95\% IC 0,53-0,82)), siendo significa tivamente superior a la warfarina en prevención de embolias asociadas a FA.

La mortalidad anual fue de $3,75 \%$ y $3,64 \%$ con dosis bajas y altas de dabigatran, respectivamente, y $4,13 \%$ con warfarina, diferencia no estadísticamente significativa.

Dabigatran 110 mg BID demostró menos sangrados que con $150 \mathrm{mg}$ BID y warfarina. Estas dos últimas presentaron incidencias similares.

Un análisis de subgrupo mostró que en pacientes con AVE transitorio (TIA) o AVE previo, ambas dosis de dabigatran disminuyeron en forma significativa los episodios de accidente cerebrovascular hemorrágico.

Si bien fue diseñado para mostrar "no inferioridad", este es el primer estudio que muestra que un fármaco supera a la warfarina en la prevención de eventos tromboembólicos en FA usando dosis altas, sin aumento significativo en los efectos $\operatorname{adversos}^{22}$.

Recientemente la FDA aprobó el uso de 150 mg BID para la prevención de eventos embólicos en $\mathrm{FA}^{23}$.

\section{Rivaroxaban}

El estudio fase 3 ROCKET-AF incluyó a más de 14.000 pacientes con FA, los cuales se asignaron en forma aleatoria a $20 \mathrm{mg}$ de rivaroxaban al día (15 mg en caso de función renal alterada) o warfarina para INR 2-3. Los pacientes tenían alto riesgo de embolia (CHADS score > 2 en 90\%). Se demostró que el nuevo anticoagulante no fue inferior a la warfarina en el criterio principal de valoración combinado, que incluyó accidente cerebrovascular y embolia sistémica. Sin embargo, al analizar con intención de tratar se demostró que no logró superioridad. 
Nuevos anticoagulantes orales - A. Berkovits et al

Tabla 2. Estudios Fase III para el tratamiento de ETE con nuevos anticoagulantes

\begin{tabular}{|llclll|}
\hline Fármaco & Control & n & Población & Outcome & Estudio \\
Dabigatran 150 mg BID & Warfarina INR 2-3 & 2.564 & TVP aguda & Dabigatran no inferior a warfarina & RE-COVER \\
$\begin{array}{l}\text { Dabigatran 110 mg o } \\
\text { 150 mg BID }\end{array}$ & Warfarina INR 2-3 & 18.113 & AC x FA & $110 \mathrm{mg}$ BID no inferior & RE-LY \\
\hline
\end{tabular}

Tabla 3. Estudios Fase III para la profilaxis de ETE con Dabigatran

\begin{tabular}{|c|c|c|c|c|}
\hline & \multicolumn{2}{|c|}{ Cirugía cadera } & \multicolumn{2}{|c|}{ Cirugía rodilla } \\
\hline & RENOVATE & RENOVATE II & REMODEL & REMOBILIZE \\
\hline $\begin{array}{l}\text { Dabigatran } \\
\text { Dosis }\end{array}$ & $\begin{array}{l}150 \text { mg po día } \\
220 \text { mg po día }\end{array}$ & 220 mg po día & $\begin{array}{l}150 \text { mg po día } \\
220 \mathrm{mg} \text { po día }\end{array}$ & $\begin{array}{l}150 \text { mg po día } \\
220 \text { mg po día }\end{array}$ \\
\hline Comparación & $\begin{array}{l}\text { Enoxaparina } \\
40 \mathrm{mg} \mathrm{SC}\end{array}$ & $\begin{array}{l}\text { Enoxaparina } \\
40 \mathrm{mg} \mathrm{SC}\end{array}$ & $\begin{array}{l}\text { Enoxaparina } \\
40 \mathrm{mg} \mathrm{SC}\end{array}$ & $\begin{array}{l}\text { Enoxaparina } \\
30 \mathrm{mg} \mathrm{SC} \text { bid }\end{array}$ \\
\hline Duración & 28-35 días & 28-35 días & 6-10 días & 12-15 días \\
\hline$n$ & 3.494 & 2.013 & 2.010 & 2.615 \\
\hline
\end{tabular}

Las tasas de hemorragia fueron similares en ambos grupos, aunque se observaron menos episodios de sangrados intracerebrales y mortales en el grupo de rivaroxaban ${ }^{24}$.

\section{Tratamiento de la enfermedad} tromboembólica venosa (Tabla 3 )

\section{Dabigatran}

El estudio RE-COVER (Study of treatment of venous thromboembolism) comparó en pacientes con primer episodio de trombosis venosa profunda (TVP) la anticoagulación con dabigatran 150 mg BID y warfarina durante 6 meses. Se demostró similar incidencia de episodios de TVP recurrente o muerte relacionada a trombosis venosa en ambos grupos.

Los sangrados mayores también fueron equivalentes, pero la frecuencia total de eventos fue significativamente menor en el grupo con dabigatran.

Se concluyó que el dabigatran tiene una eficacia comparable con la warfarina y que tendría menos efectos adversos. Por tanto, podría constituir una alternativa oral, con dosis fija y sin control de laboratorio para el tratamiento de enfermedad tromboembólica ${ }^{25}$.

\section{Rivaroxaban}

Los estudios, EINSTEIN-DVT, EINSTEIN-PE evaluaron 2.900 pacientes con episodio de TVP, sin tromboembolismo pulmonar (TEP) y 3.300 con TEP respectivamente. Se comparó warfarina en rangos terapéuticos (INR 2-3) y rivaroxaban como tratamiento de enfermedad tromboembólica venosa por 3,6 y 12 meses según la indicación del médico tratante. Los objetivos principales fueron recurrencia de TVP y muerte asociada a esta recurrencia. Los objetivos de seguridad incluían sangrados, infartos al miocardio, elevación de transaminasas o cualquier otro efecto adverso reportado.

Los resultados del EINSTEIN-DVT mostraron incidencia de objetivo primario en 2,1\% para dabigatran y $3 \%$ para warfarina, con significación para no inferioridad (95\% CI, 0,11-1,04; 0 $<0,001)$. El objetivo de seguridad ocurrió en $8,1 \%$ en cada grupo.

El estudio EINSTEIN-PE aún no ha sido publicado.

Una vez finalizado el tratamiento anticoagulante se evaluó extender la profilaxis secundaria con rivaroxaban $20 \mathrm{mg} /$ día comparado con placebo por 6-12 meses (EINSTEIN-EXT). Rivaroxaban mostró una reducción del riesgo relativo del objetivo primario en $88 \%$, sin diferencias en sangrado $^{26}$.

Este hallazgo es altamente promisorio, considerando que la recurrencia promedio de un paciente 
Tabla 4. Estudios Fase III para la profilaxis de ETE con Rivaroxaban

\begin{tabular}{|lllll|}
\hline & Cadera & & Rodilla & \\
& RECORD 1 & RECORD 2 & RECORD 3 & RECORD 4 \\
Rivaroxaban & $10 \mathrm{mg}$ día & $10 \mathrm{mg}$ día & $10 \mathrm{mg}$ día & 10 mg día \\
Comparación & $\begin{array}{l}\text { Enoxaparina } 40 \mathrm{mg} \\
\text { SC día }\end{array}$ & $\begin{array}{l}\text { Enoxaparina } 40 \mathrm{mg} \\
\text { SC día }\end{array}$ & $\begin{array}{l}\text { Enoxaparina } 40 \mathrm{mg} \\
\text { SC día }\end{array}$ & $\begin{array}{l}\text { Enoxaparina } 30 \mathrm{mg} \\
\text { SC bid }\end{array}$ \\
Duración & $\begin{array}{l}\text { Riva:35 días } \\
\text { Enox: } 35 \text { días }\end{array}$ & $\begin{array}{l}\text { Riva:35 días } \\
\text { Enox:14 días }\end{array}$ & 14 días & 14 días \\
$\mathrm{n}$ & 4.541 & 2.509 & 2.531 & 3.148 \\
\hline
\end{tabular}

tratado por 12 meses con terapia anticoagulante es de aproximadamente $10 \%$ el primer año, mostrando seguridad en prolongación de anticoagulación.

\section{Profilaxis primaria TVP (Tablas 4 y 5)}

Definimos a la profilaxis primaria de TVP como las medidas farmacológicas utilizadas para prevenir la aparición de una trombosis venosa profunda en pacientes que no la han presentado.

\section{Dabigatran}

Los estudios RE-NOVATE I y II, compararon el dabigatran en dosis de 150 mg y 220 mg y sólo 220 mg una vez al día (QD), respectivamente, y enoxaparina por 28 a 35 días en pacientes sometidos a reemplazo total de cadera. El objetivo primario fue evaluar la incidencia de eventos tromboembólicos y la mortalidad. No hubo diferencias significativas en ambos objetivos ni en episodios de sangrado.

Dabigatran mostró ser "no inferior" a enoxaparina en la prevención de TVP/TEP en pacientes sometidos a cirugía de reemplazo total de cadera, sin aumentar los episodios de sangrado ${ }^{27}$.

El Estudio RE-MODEL comparó dabigatran en dos dosis: 150 mg y 220 mg QD con enoxaparina $40 \mathrm{mg} /$ día en pacientes sometidos a artroplastía de rodilla. El objetivo primario fue evaluar la incidencia de episodios tromboembólicos y la mortalidad. Los resultados no mostraron diferencias entre ambos fármacos, ni en episodios de sangrado, concluyendo que el dabigatran sería tan efectivo como la enoxaparina para la prevención de fenómenos tromboembólicos y muerte en cirugía de rodilla ${ }^{28}$.

El estudio RE-MOBILIZE evaluó en forma similar dabigatran en las dosis antes mencionadas, pero ahora con enoxaparina $30 \mathrm{mg}$ BID. En este estudio el dabigatran fue inferior a la enoxaparina en prevención de eventos tromboembólicos. Cabe destacar que las dosis utilizadas de enoxaparina superan a las habituales en profilaxis para trombosis venosa profunda ${ }^{29}$.

En septiembre de 2010 se analizaron en conjunto tres de los cuatro trabajos antes mencionados, concluyendo que el dabigatran es tan efectivo como la enoxaparina en la prevención de eventos tromboembólicos en pacientes sometidos a cirugía traumatológica de extremidades inferiores, sin aumentar los episodios de sangrado ${ }^{30}$.

\section{Rivaroxaban}

El estudio RECORD 1 evaluó la efectividad del rivaroxaban en la prevención de la enfermedad tromboembólica, en pacientes sometidos a artroplastía total de cadera. Se sometieron 4.541 pacientes a selección aleatoria para recibir rivaroxaban 10 mg QD o enoxaparina $40 \mathrm{mg}$ QD hasta $35 \pm 4$ días post cirugía. Se evaluaron los resultados $36 \pm$ 4 días posterior a la última dosis de anticoagulante. El objetivo primario fue evaluar la incidencia de episodios de tromboembolismo venoso o muerte. Se observó una reducción absoluta (RRA) del objetivo primario en 2,6\% con el inhibidor de FXa en comparación con enoxaparina $(-2,6 \%$, CI 95\% $(-3,7 \%$ a $1,5 \%) \mathrm{p}<0,001)$ con una reducción del riesgo relativa (RRR) de 70\%, explicado principalmente por disminución en episodios de tromboembolismo sin modificación en la mortalidad. No hubo diferencias en los episodios de sangrado ${ }^{31}$.

El estudio RECORD 2 es similar al RECORD 1 , pero con la dosis de enoxaparina administrada sólo por 12 días y luego continuación con placebo, sin modificación en la administración de rivaroxa- 
ban, mostrando resultados similares con RRR de $79 \%$ en episodios de tromboembolismo ${ }^{32}$.

El estudio RECORD 3 comparó rivaroxaban $10 \mathrm{mg}$ QD y enoxaparina $40 \mathrm{mg}$ QD por $12 \pm 2$ días en pacientes sometidos a reemplazo total de rodilla, mostrando reducción significativa de los episodios de trombosis venosa distales, pero sin diferencias en trombosis proximales, tromboembolismo pulmonar o mortalidad ${ }^{33}$.

El estudio RECORD 4 es similar al RECORD 3, pero comparando rivaroxaban $10 \mathrm{mg}$ QD y enoxaparina $30 \mathrm{mg}$ BID por 14 días. Rivaroxaban reduce significativamente los episodios de tromboembolismo venoso en ambas comparaciones, con RRR 31\% respectivamente. Los episodios de sangrado fueron similares en los tres grupos ${ }^{34}$.

Se concluye de los estudios RECORD que el rivaroxaban es superior a la enoxaparina en la prevención del tromboembolismo venoso, en pacientes sometidos a cirugías traumatológicas de rodilla y cadera.

Se encuentra en curso un ensayo clínico fase III para evaluar el rivaroxaban en la prevención del TVP, en pacientes médicos de alto riesgo, comparando $10 \mathrm{mg}$ QD por 35 días con enoxaparina 40 mg QD por $10 \pm 4$ días. (Estudio MAGELLAN).

\section{Apixaban}

El estudio ADVANCE-1 en reemplazo total de rodilla comparó apibaxan 2,5 mg BID y enoxaparina $30 \mathrm{mg}$ BID por $12 \pm 2$ días. Si bien los resultados no muestran diferencias significativas de eventos trombóticos y muerte, en este estudio, el apixaban no alcanzó los criterios de no inferioridad frente a la enoxaparina. Destaca, eso sí, la disminución de sangrados mayores clínicamente relevantes y el uso de una dosis mayor de heparinas de bajo peso molecular ${ }^{35}$.

El estudio ADVANCE-2 comparó apixaban 2,5 mg BID y enoxaparina $40 \mathrm{mg}$ QD en el mismo grupo de pacientes explicitados en el estudio. Se observó una reducción significativa de los eventos tromboembólicos con apixaban (RRR de 38\%), sin diferencias en riesgo de sangrado ${ }^{35}$.

\section{Conclusiones}

Las nuevas drogas anticoagulantes orales están mostrando tener una serie de ventajas de uso, respecto a los antagonistas de la vitamina $\mathrm{K}$.
Los ensayos clínicos disponibles muestran que estas nuevas drogas son al menos tan efectivas y seguras como los cumarínicos en el manejo de las trombosis, tanto arterial como venosa.

La conclusión definitiva sobre su seguridad y efectividad se obtendrá una vez concluidos la serie de ensayos clínicos fase III y la evaluación de drogas ya aprobadas en ensayos de vigilancia fase IV. Sin embargo, aunque la evidencia es una herramienta de gran utilidad, tenemos que esperar la aparición de mayores estudios clínicos en distintas poblaciones y realidades, para así comprobar el real beneficio de estas drogas.

Los problemas de estos nuevos fármacos son que carecen de métodos demostrados de monitorización, por lo que en pacientes con características disímiles (como sobre o bajo peso, insuficiencia renal o hepática) la "dosis fija” puede no resultar terapéutica. No existen aún estudios que avalen el uso de algún antídoto en caso de sobredosificación con sangrado. Por último, sus costos son muy superiores a los de los anticoagulantes tradicionales.

\section{Referencias}

1. Last JA. The Missing Link: The Story of Karl Paul Link. Toxicol Sci 2002; 66: 4-6.

2. Whitlon DS, Sadowski JA, Suttie JW. Mechanism of coumarin action: significance of vitamin $\mathrm{K}$ epoxide reductase inhibition. Biochemistry 1978; 17: 1371-7.

3. Stehle S, Kirchheiner J, Lazar A, Fuhr U. Pharmacogenetics of oral anticoagulants: a basis for dose individualization. Clin Pharmacokinet 2008; 47: 565-94.

4. Ahrens I, Lip GY, Peter K. New oral anticoagulant drugs in cardiovascular disease. Thromb Haemost 2010; 104: 49-60.

5. Hanssong K. Inflammation, Atherosclerosis, and Coronary Artery Disease. N Engl J Med 2005; 352: 1685-95.

6. Defunciones según grupo de edad, por servicio de salud, gran grupo de causas de muertes y sexo, 2009. Departamento de estadísticas. MINSAL, Chile.

7. Silverstein MD, Heit JA, Mohr DN, Petterson TM, O'Fallon WM, Melton LJ $3^{\text {rd }}$. Risk factors for deep vein thrombosis and pulmonary embolism: a populationbased case-control study. Arch Intern Med 2000; 160: 809-15.

8. Wood JP, Silveira JR, Maille NM, Haynes LM, Tracy PB. Prothrombin activation on the activated platelet surface optimizes expression of procoagulant activity. Blood 2011; 117: 1710-8. 
9. Hoffman M, Monroe DM $3^{\text {rd }}$. A cell-based model of hemostasis. Thromb Haemost 2001; 85: 958-65.

10. Panes O, Matus V, Sáez CG, Quiroga T, Pereira J, Mezzano D. Human platelets synthesize and express functional tissue factor. Blood 2007; 109: 5242-50.

11. Hoffman M, Monroe DM. Coagulation 2006: a modern view of hemostasis. Hematol Oncol Clin North Am 2007; 21: 1-11.

12. Kretz CA, Vaezzadeh N, Gross PL. Tissue factor and thrombosis models. Arterioscler Thromb Vasc Biol 2010; 30: 900-8.

13. Whitlon DS, Sadowski JA, Suttie JW. Mechanism of coumarin action: significance of vitamin $\mathrm{K}$ epoxide reductase inhibition. Biochemistry 1978; 17: 1371-7.

14. Wallin R, Hutson SM. Warfarin and the vitamin Kdependent gamma-carboxylation system. Trends Mol Med 2004; 10: 299-302.

15. Manolopoulos VG, Ragia G, Tavridou A. Pharmacogenetics of coumarinic oral anticoagulants. Pharmacogenomics 2010; 11: 493-6.

16. Ahrens I, Lip GY, Peter K.. New oral anticoagulant drugs in cardiovascular disease. Thromb Haemost 2010; 104: 49-60.

17. Eisert WG, Hauel N, Stangier J, Wienen W, Clemens A, van Ryn J. Dabigatran: an oral novel potent reversible nonpeptide inhibitor of thrombin. Arterioscler Thromb Vasc Biol 2010; 30: 1885-9.

18. Bovio JA, Smith SM, Gums JG. Dabigatran etexilate: a novel oral thrombin inhibitor for thromboembolic disease. Ann Pharmacother 2011; 45: 603-14.

19. Mousa SA. Oral direct factor Xa inhibitors, with special emphasis on Rivaroxaban. Methods Mol Biol 2010; 663: 181-201.

20. Raghavan N, Frost CE, Yu Z, He K, Zhang H, Humphreys WG, et al. Apixaban metabolism and pharmacokinetics after oral administration to humans. Drug Metab Dispos 2009; 37: 74-81.

21. Ellis DJ, Usman MH, Milner PG, Canafax DM, Ezekowitz MD. The First Evaluation of a Novel Vitamin K Antagonist, Tecarfarin (ATI-5923), in Patients With Atrial Fibrillation. Circulation 2009; 120: 1029-35.

22. Connolly SJ, Ezekowitz MD, Yusuf S, Eikelboom J, Oldgren J, Parekh A, et al. Dabigatran versus warfarina in patients with atrial fibrillation. N Engl J Med 2009; 361: 1139-51.

23. Beasley BN, Unger EF, Temple R. Anticoagulant Options-Why the FDA Approved a Higher but Not a Lower Dose of Dabigatran. N Engl J Med 2011; 364: 1788-90.

24. ROCKET AF Study Investigators. Rivaroxaban-once daily, oral, direct factor Xa inhibition compared with vitamin $\mathrm{K}$ antagonism for prevention of stroke and Embolism Trial in Atrial Fibrillation: rationale and design of the ROCKET AF study. Am Heart J 2010; 159: 340-7.

25. Schulman S, Kearon C, Kakkar AK, Mismetti P, Schellong S, Eriksson H, et al. Dabigatran versus warfarin in the treatment of acute venous thromboembolism. N Engl J Med 2009; 361: 2342-52.

26. Bauersachs R, Berkowitz SD, Brenner B, Buller HR, Decousus H, Gallus AS, et al. Oral rivaroxaban for symptomatic venous thromboembolism. N Engl J Med 2010; 363: 2499-510.

27. Eriksson BI, Dahl OE, Rosencher N, Kurth AA, van Dijk $\mathrm{CN}$, Frostick SP. Dabigatran etexilate versus enoxaparin for prevention of venous thromboembolism after total hip replacement: a randomised, double-blind, noninferiority trial. Lancet 2007; 370: 949-56.

28. Eriksson BI, Dahl OE, Rosencher N, Kurth AA, van Dijk CN, Frostick SP, et al. Oral dabigatran etexilate vs. subcutaneous enoxaparin for the prevention of venous thromboembolism after total knee replacement: the REMODEL randomized trial. J Thromb Haemost 2007; 11: 2178-85.

29. Ginsberg JS, Davidson BL, Comp PC, Francis CW, Friedman RJ, Huo MH, et al. Oral thrombin inhibitor dabigatran etexilate vs North American enoxaparin regimen for prevention of venous thromboembolism after knee arthroplasty surgery. J Arthroplasty 2009; 24: 1-9.

30. Friedman RJ, Dahl OE, Rosencher N, Caprini JA, Kurth AA, Francis CW, et al. Dabigatran versus enoxaparin for prevention of venous thromboembolism after hip or knee arthroplasty: a pooled analysis of three trials. Thromb Res 2010; 126: 175-82.

31. Eriksson BI, Borris LC, Friedman RJ, Haas S, Huisman MV, Kakkar AK, et al. Rivaroxaban versus enoxaparin for thromboprophylaxis after hip arthroplasty. N Engl J Med 2008; 358: 2765-75.

32. Kakkar AK, Brenner B, Dahl OE, Eriksson BI, Mouret $\mathrm{P}$, Muntz J, et al. Extended duration Rivaroxaban versus short-term enoxaparin for the prevention of venous thromboembolism after total hip arthroplasty: a doubleblind, randomised controlled trial. Lancet 2008; 372: 31-9.

33. Lassen MR, Ageno W, Borris LC, Lieberman JR, Rosencher N, Bandel TJ, et al. Rivaroxaban versus enoxaparin for thromboprophylaxis after total knee arthroplasty. N Engl J Med 2008; 358: 2776-86.

34. Turpie AG, Lassen MR, Davidson BL, Bauer KA, Gent M, Kwong LM, et al. Rivaroxaban versus enoxaparin for thromboprophylaxis after total knee arthroplasty (RE- 
Nuevos anticoagulantes orales - A. Berkovits et al

CORD4): a randomised trial. Lancet 2009; 373: 1673-80.

35. Lassen MR, Raskob GE, Gallus A, Pineo G, Chen D, Portman RJ. Apixaban or Enoxaparin for thromboprophylaxis after knee replacement. N Engl J Med 2009; 361: 594-604.
36. Lassen MR, Raskob GE, Gallus A, Pineo G, Chen D, Hornick P. Apixaban versus Enoxaparin for thromboprophylaxis after knee replacement (ADVANCE-2): a randomised double-blind trial. Lancet 2010; 375: 807- 